\title{
Convergence rates in the law of large numbers for long-range dependent linear processes
}

\author{
Tao Zhang ${ }^{1}$, Pingyan Chen² and Soo Hak Sung ${ }^{3 *}$ (D)
}

\section{"Correspondence:}

sungsh@pcu.ac.kr

${ }^{3}$ Department of Applied

Mathematics, Pai Chai University,

Daejeon, 35345, South Korea

Full list of author information is

available at the end of the article

\begin{abstract}
Baum and Katz (Trans. Am. Math. Soc. 120:108-123, 1965) obtained convergence rates in the Marcinkiewicz-Zygmund law of large numbers. Their result has already been extended to the short-range dependent linear processes by many authors. In this paper, we extend the result of Baum and Katz to the long-range dependent linear processes. As a corollary, we obtain convergence rates in the Marcinkiewicz-Zygmund law of large numbers for short-range dependent linear processes.
\end{abstract}

MSC: $60 \mathrm{~F} 15$

Keywords: linear process; convergence rate; Marcinkiewicz-Zygmund law of large numbers

\section{Introduction}

There are many literature works concerning the convergence rates in the MarcinkiewiczZygmund law of large numbers. One can refer to Alf [2], Alsmeyer [3], Baum and Katz [1], Heyde and Rohatgi [4], Hu and Weber [5], Rohatgi [6], and so on.

Baum and Katz [1] obtained the following convergence rates in the MarcinkiewiczZygmund law of large numbers.

Theorem 1.1 (Baum and Katz [1]) Let $r \geq 1,1 \leq p<2$ and $\left\{X, X_{n}, n \geq 1\right\}$ be a sequence of independent and identically distributed (i.i.d.) random variables. Then $E X=0$ and $E|X|^{r p}<\infty$ imply

$$
\sum_{n=1}^{\infty} n^{r-2} P\left(\left|\sum_{k=1}^{n} X_{k}\right|>n^{1 / p} \varepsilon\right)<\infty \quad \text { for all } \varepsilon>0 .
$$

When $r=2$, the cases of $p=1$ and $1 \leq p<2$ have already been proved by Hsu and Robbins [7] and Katz [8], respectively.

Let $\left\{\zeta_{i}, i \in \mathbb{Z}\right\}$ be a sequence of i.i.d. random variables and $\left\{a_{i}, i \in \mathbb{Z}\right\}$ be a sequence of real numbers. Here and in the following, $\mathbb{Z}$ denotes the set of all integers. Then $\left\{X_{n}, n \geq 1\right\}$ is 
called a linear process or an infinite order moving average process if $X_{n}$ is defined by

$$
X_{n}=\sum_{i=-\infty}^{\infty} a_{i+n} \zeta_{i} \quad \text { for } n \geq 1
$$

If $\sum_{i=-\infty}^{\infty}\left|a_{i}\right|<\infty$, then $\left\{X_{n}, n \geq 1\right\}$ has short memory or is short-range dependent. If $\sum_{i=-\infty}^{\infty}\left|a_{i}\right|=\infty$, then $\left\{X_{n}, n \geq 1\right\}$ has long memory or is long-range dependent (see Chapter 3 in Giraitis et al. [9]).

In the short-range dependent case, Koopmans [10] showed that if $\zeta_{0}$ has the moment generating function, then the strong law of large numbers for the linear process holds with exponential convergence rate. Hanson and Koopmans [11] generalized this result to a class of linear processes of independent but non-identically distributed random variables $\left\{\zeta_{i}, i \in \mathbb{Z}\right\}$ and to arbitrary subsequences of $\left\{X_{n}, n \geq 1\right\}$. Li et al. [12] extended Katz [8] theorem to the setting of short-range dependent linear processes.

Theorem 1.2 (Li et al. [12]) Let $1 \leq p<2$. Let $\left\{a_{i}, i \in \mathbb{Z}\right\}$ be an absolutely summable sequence of real numbers. Suppose that $\left\{X_{n}, n \geq 1\right\}$ is the linear process of a sequence $\left\{\zeta_{i}, i \in \mathbb{Z}\right\}$ of i.i.d. random variables with mean zero and $E\left|\zeta_{0}\right|^{2 p}<\infty$. Then

$$
\sum_{n=1}^{\infty} P\left(\left|\sum_{k=1}^{n} X_{k}\right|>n^{1 / p} \varepsilon\right)<\infty \text { for all } \varepsilon>0 .
$$

Note that Theorem 1.2 corresponds to Theorem 1.1 with $r=2$. Zhang [13] extended Theorem 1.1 with $r>1$ to the short-range dependent linear process of a sequence of identically distributed $\varphi$-mixing random variables. Since independent random variables are also $\varphi$-mixing, it follows by Zhang [13] theorem that Theorem 1.2 also holds for $r>1$.

In this paper, we obtain convergence rates in the Marcinkiewicz-Zygmund law of large numbers for long-range dependent linear processes of i.i.d. random variables. For convenience of notation, let

$$
W_{n}(t)=\left(\sum_{i=-\infty}^{\infty}\left|\omega_{n i}\right|^{t}\right)^{1 / t} \quad \text { for } n \geq 1 \text { and } t>0,
$$

where $\omega_{n i}=\sum_{k=1}^{n} a_{i+k}$. In the long-range dependent case, Characiejus and Račkauskas [14] obtained the convergence rate in the Marcinkiewicz-Zygmund law of large numbers for the linear process $\left\{Y_{n}, n \geq 1\right\}$ which is slightly different from (1.1) and defined by

$$
Y_{n}=\sum_{i=0}^{\infty} a_{i} \zeta_{n-i} \quad \text { for } n \geq 1,
$$

where $a_{i}=0$ if $i<0$.

Theorem 1.3 (Characiejus and Račkauskas [14]) Let $\left\{Y_{n}, n \geq 1\right\}$ be defined as above and $1<p<2$. Let $\left\{a_{i}, i \in \mathbb{Z}\right\}$ be a sequence of real numbers such that

$$
\sum_{i=-\infty}^{\infty}\left|a_{i}\right|^{p}<\infty
$$


where $a_{i}=0$ if $i<0$. Assume that

$$
\begin{gathered}
W_{n}(q) / W_{n}(p)=O\left(n^{1 / q-1 / p}\right) \quad \text { for some } q \in(p, 2] . \\
\text { If } E \zeta_{0}=0 \text { and } E\left[\left|\zeta_{0}\right|^{p} \log \left(1+\left|\zeta_{0}\right|\right)\right]<\infty \text {, then } \\
\sum_{n=1}^{\infty} n^{-1} P\left(\left|\sum_{k=1}^{n} Y_{k}\right|>W_{n}(p) \varepsilon\right)<\infty \text { for all } \varepsilon>0 .
\end{gathered}
$$

The above theorem shows a convergence rate in the Marcinkiewicz-Zygmund weak law of large numbers with the norming sequence $W_{n}(p)$.

We now compare Theorem 1.3 with Theorem 1.1. Since Theorem 1.3 deals with only the case $r=1$, it is interesting to prove that Theorem 1.3 holds for the case $r>1$. When $r=1$, Theorem 1.1 requires a finite $p$ th moment condition, but Theorem 1.3 requires more than finite $p$ th moment. To apply Theorem 1.3 , it is necessary to estimate $W_{n}(p)$. If $\left\{a_{i}, i \in \mathbb{Z}\right\}$ is an absolutely summable sequence, then we have, by the result of Burton and Dehling [15] (see also Lemma 2.4), that for any $t>0$

$$
\frac{1}{n} W_{n}^{t}(t) \rightarrow \sum_{i=-\infty}^{\infty} a_{i}
$$

and hence (1.3) holds with $W_{n}(p)$ replaced by $n^{1 / p}$. However, for the long-range dependent case, it is not easy to estimate $W_{n}(t)$.

In this paper, we extend Theorem 1.1 to the long-range dependent linear processes. As a corollary, we obtain a long-range dependent setting of Theorem 1.2. Further, we propose a method to estimate $W_{n}(t)$ for the long-range dependent case.

Throughout this paper, $C$ denotes a positive constant which may vary at each occurrence. For events $A$ and $B, I(A)$ denotes the indicator function of the event $A$, and $I(A, B)=I(A \cap B)$.

\section{Convergence of long-range dependent linear processes}

In this section, we extend Theorem 1.1 to the long-range dependent linear processes. To prove the main results, we need the following lemmas. The first one is the von Bahr-Esseen inequality (see von Bahr and Esseen [16]). The second is known as Fuk-Nagaev inequality (see Corollary 1.8 in Nagaev [17]).

Lemma 2.1 Let $\left\{\zeta_{i}, i \geq 1\right\}$ be a sequence of independent random variables with $E \zeta_{i}=0$ and $E\left|\zeta_{i}\right|^{t}<\infty$ for some $1 \leq t \leq 2$. Then, for all $n \geq 1$,

$$
E\left|\sum_{i=1}^{n} \zeta_{i}\right|^{t} \leq C_{t} \sum_{i=1}^{n} E\left|\zeta_{i}\right|^{t}
$$

where $C_{t}>0$ is a positive constant depending only on $t$. 
Lemma 2.2 Let $\left\{\zeta_{i}, i \geq 1\right\}$ be a sequence of independent random variables with $E \zeta_{i}=0$. Then, for any $t \geq 2$ and $x>0$,

$$
P\left(\left|\sum_{i=1}^{n} \zeta_{i}\right|>x\right) \leq(1+2 / t)^{t} x^{-t} \sum_{i=1}^{n} E\left|\zeta_{i}\right|^{t}+2 \exp \left\{-\frac{2 x^{2}}{(t+2)^{2} e^{t} \sum_{i=1}^{n} \operatorname{Var}\left(\zeta_{i}\right)}\right\} .
$$

The following lemma is well known and can be easily proved by using a standard method.

Lemma 2.3 Let $p>0$ and $\zeta$ be a random variable. Then the following statements hold.

(i) If $0<\theta<p$, then $\sum_{n=1}^{\infty} n^{-\theta / p} E|\zeta|^{\theta} I\left(|\zeta|>n^{1 / p}\right) \leq C E|\zeta|^{p}$.

(ii) If $p<q$, then $\sum_{n=1}^{\infty} n^{-q / p} E|\zeta|^{q} I\left(|\zeta| \leq n^{1 / p}\right) \leq C E|\zeta|^{p}$.

(iii) If $r>1$, then $\sum_{n=1}^{\infty} n^{r-2} E|\zeta|^{p} I\left(|\zeta|>n^{1 / p}\right) \leq C E|\zeta|^{r p}$.

(iv) If $r p<q$, then $\sum_{n=1}^{\infty} n^{r-1-q / p} E|\zeta|^{q} I\left(|\zeta| \leq n^{1 / p}\right) \leq C E|\zeta|^{r p}$.

The following lemma is useful to estimate $W_{n}(t)$ when the sequence $\left\{a_{i}, i \in \mathbb{Z}\right\}$ is absolutely summable. However, it is not applicable to the long-range dependent case.

Lemma 2.4 (Burton and Dehling [15]) Let $\sum_{i=-\infty}^{\infty} a_{i}$ be an absolutely convergent series of real numbers with $a=\sum_{i=-\infty}^{\infty} a_{i}$. Then, for any $t>0$,

$$
\lim _{n \rightarrow \infty} \frac{1}{n} \sum_{i=-\infty}^{\infty}\left|\omega_{n i}\right|^{t}=|a|^{t}
$$

where $\omega_{n i}=\sum_{k=1}^{n} a_{i+k}$.

We now state and prove our main results. The first theorem treats the case $r>1$.

Theorem 2.1 Let $r>1$ and $1 \leq p<2$. Let $\left\{a_{i}, i \in \mathbb{Z}\right\}$ be a sequence of real numbers with

$$
\sum_{i=-\infty}^{\infty}\left|a_{i}\right|^{p}<\infty
$$

Suppose that $\left\{X_{n}, n \geq 1\right\}$ is the linear process of a sequence $\left\{\zeta_{i}, i \in \mathbb{Z}\right\}$ of i.i.d. random variables with mean zero and $E\left|\zeta_{0}\right|^{r p}<\infty$. Furthermore, assume that one of the following conditions holds.

(1) If $1<r p<2$, then

$$
W_{n}(q) / W_{n}(p)=O\left(n^{1 / q-1 / p}\right) \quad \text { for some } q \in(r p, 2) .
$$

(2) If $r p \geq 2$, then

$$
W_{n}(q) / W_{n}(p)=O\left(n^{1 / q-1 / p}\right) \text { for some } q>r p
$$

and

$$
W_{n}(s) / W_{n}(p)=o\left((\log n)^{-1 / s}\right) \quad \text { for some } s \in(p, 2] \text {. }
$$


Then

$$
\sum_{n=1}^{\infty} n^{r-2} P\left(\left|\sum_{k=1}^{n} X_{k}\right|>W_{n}(p) \varepsilon\right)<\infty \text { for all } \varepsilon>0 .
$$

Proof (1) For each $n \geq 1$, we have

$$
\begin{aligned}
\sum_{k=1}^{n} X_{k}= & \sum_{i=-\infty}^{\infty} \sum_{k=1}^{n} a_{i+k} \zeta_{i}=\sum_{i=-\infty}^{\infty} \omega_{n i} \zeta_{i} \\
= & \sum_{i=-\infty}^{\infty} \omega_{n i}\left[\zeta_{i} I\left(\left|\zeta_{i}\right|>n^{1 / p}\right)-E \zeta_{i} I\left(\left|\zeta_{i}\right|>n^{1 / p}\right)\right] \\
& +\sum_{i=-\infty}^{\infty} \omega_{n i}\left[\zeta_{i} I\left(\left|\zeta_{i}\right| \leq n^{1 / p}\right)-E \zeta_{i} I\left(\left|\zeta_{i}\right| \leq n^{1 / p}\right)\right] \\
:= & S_{n}^{\prime}+S_{n}^{\prime \prime}
\end{aligned}
$$

and hence,

$$
\begin{aligned}
& \sum_{n=1}^{\infty} n^{r-2} P\left(\left|\sum_{k=1}^{n} X_{k}\right|>W_{n}(p) \varepsilon\right) \\
& \leq \sum_{n=1}^{\infty} n^{r-2} P\left(\left|S_{n}^{\prime}\right|>W_{n}(p) \varepsilon / 2\right)+\sum_{n=1}^{\infty} n^{r-2} P\left(\left|S_{n}^{\prime \prime}\right|>W_{n}(p) \varepsilon / 2\right) .
\end{aligned}
$$

By the Markov inequality, Lemmas 2.1 and 2.3, we have

$$
\begin{aligned}
\sum_{n=1}^{\infty} n^{r-2} P\left(\left|S_{n}^{\prime}\right|>W_{n}(p) \varepsilon / 2\right) & \leq \sum_{n=1}^{\infty} n^{r-2} \frac{2^{p} E\left|S_{n}^{\prime}\right|^{p}}{\varepsilon^{p} W_{n}^{p}(p)} \\
& \leq C \sum_{n=1}^{\infty} n^{r-2} \frac{\sum_{i=-\infty}^{\infty}\left|\omega_{n i}\right|^{p} E\left|\zeta_{0}\right|^{p} I\left(\left|\zeta_{0}\right|>n^{1 / p}\right)}{\sum_{i=-\infty}^{\infty}\left|\omega_{n i}\right|^{p}} \\
& =C \sum_{n=1}^{\infty} n^{r-2} E\left|\zeta_{0}\right|^{p} I\left(\left|\zeta_{0}\right|>n^{1 / p}\right) \\
& \leq C E\left|\zeta_{0}\right|^{r p}<\infty .
\end{aligned}
$$

Thus the first series on the right-hand side of (2.1) converges.

Similarly, by the Markov inequality, Lemmas 2.1 and 2.3, we have

$$
\begin{aligned}
\sum_{n=1}^{\infty} n^{r-2} P\left(\left|S_{n}^{\prime \prime}\right|>W_{n}(p) \varepsilon / 2\right) & \leq \sum_{n=1}^{\infty} n^{r-2} \frac{2^{q} E\left|S_{n}^{\prime \prime}\right|^{q}}{\varepsilon^{q} W_{n}^{q}(p)} \\
& \leq C \sum_{n=1}^{\infty} n^{r-2} \frac{\sum_{i=-\infty}^{\infty}\left|\omega_{n i}\right|^{q} E\left|\zeta_{0}\right|^{q} I\left(\left|\zeta_{0}\right| \leq n^{1 / p}\right)}{W_{n}^{q}(p)} \\
& =C \sum_{n=1}^{\infty} n^{r-2}\left(\frac{W_{n}(q)}{W_{n}(p)}\right)^{q} E\left|\zeta_{0}\right|^{q} I\left(\left|\zeta_{0}\right| \leq n^{1 / p}\right)
\end{aligned}
$$




$$
\begin{aligned}
& \leq C \sum_{n=1}^{\infty} n^{r-2}\left(n^{1 / q-1 / p}\right)^{q} E\left|\zeta_{0}\right|^{q} I\left(\left|\zeta_{0}\right| \leq n^{1 / p}\right) \\
& =C \sum_{n=1}^{\infty} n^{r-1-q / p} E\left|\zeta_{0}\right|^{q} I\left(\left|\zeta_{0}\right| \leq n^{1 / p}\right) \\
& \leq C E\left|\zeta_{0}\right|^{r p}<\infty
\end{aligned}
$$

Hence the second series on the right-hand side of (2.1) also converges.

(2) For each $n \geq 1$, we have

$$
\begin{aligned}
\sum_{k=1}^{n} X_{k}= & \sum_{i=-\infty}^{\infty} \sum_{k=1}^{n} a_{i+k} \zeta_{i}=\sum_{i=-\infty}^{\infty} \omega_{n i} \zeta_{i} \\
= & \sum_{i=-\infty}^{\infty}\left[\omega_{n i} \zeta_{i} I\left(\left|\omega_{n i} \zeta_{i}\right|>W_{n}(p)\right)-E \omega_{n i} \zeta_{i} I\left(\left|\omega_{n i} \zeta_{i}\right|>W_{n}(p)\right)\right] \\
& +\sum_{i=-\infty}^{\infty}\left[\omega_{n i} \zeta_{i} I\left(\left|\omega_{n i} \zeta_{i}\right| \leq W_{n}(p)\right)-E \omega_{n i} \zeta_{i} I\left(\left|\omega_{n i} \zeta_{i}\right| \leq W_{n}(p)\right)\right] \\
:= & T_{n}^{\prime}+T_{n}^{\prime \prime}
\end{aligned}
$$

and hence,

$$
\begin{aligned}
& \sum_{n=1}^{\infty} n^{r-2} P\left(\left|\sum_{k=1}^{n} X_{k}\right|>W_{n}(p) \varepsilon\right) \\
& \quad \leq \sum_{n=1}^{\infty} n^{r-2} P\left(\left|T_{n}^{\prime}\right|>W_{n}(p) \varepsilon / 2\right)+\sum_{n=1}^{\infty} n^{r-2} P\left(\left|T_{n}^{\prime \prime}\right|>W_{n}(p) \varepsilon / 2\right) .
\end{aligned}
$$

By the Markov inequality, Lemmas 2.1 and 2.3, we have

$$
\begin{aligned}
\sum_{n=1}^{\infty} & n^{r-2} P\left(\left|T_{n}^{\prime}\right|>W_{n}(p) \varepsilon / 2\right) \\
\leq & \sum_{n=1}^{\infty} n^{r-2} \frac{2^{p} E\left|T_{n}^{\prime}\right|^{p}}{\varepsilon^{p} W_{n}^{p}(p)} \\
\leq & C \sum_{n=1}^{\infty} n^{r-2} \frac{\sum_{i=-\infty}^{\infty} E\left|\omega_{n i} \zeta_{i}\right|^{p} I\left(\left|\omega_{n i} \zeta_{i}\right|>W_{n}(p)\right)}{W_{n}^{p}(p)} \\
= & C \sum_{n=1}^{\infty} n^{r-2} \frac{\sum_{i=-\infty}^{\infty} E\left|\omega_{n i} \zeta_{i}\right|^{p} I\left(\left|\omega_{n i} \zeta_{i}\right|>W_{n}(p),\left|\zeta_{i}\right|>n^{1 / p}\right)}{W_{n}^{p}(p)} \\
& +C \sum_{n=1}^{\infty} n^{r-2} \frac{\sum_{i=-\infty}^{\infty} E\left|\omega_{n i} \zeta_{i}\right|^{p} I\left(\left|\omega_{n i} \zeta_{i}\right|>W_{n}(p),\left|\zeta_{i}\right| \leq n^{1 / p}\right)}{W_{n}^{p}(p)} \\
\leq & C \sum_{n=1}^{\infty} n^{r-2} \frac{\sum_{i=-\infty}^{\infty}\left|\omega_{n i}\right|^{p} E\left|\zeta_{i}\right|^{p} I\left(\left|\zeta_{i}\right|>n^{1 / p}\right)}{W_{n}^{p}(p)} \\
& +C \sum_{n=1}^{\infty} n^{r-2} \frac{\sum_{i=-\infty}^{\infty} E\left[\left|\omega_{n i} \zeta_{i}\right|^{p-q}\left|\omega_{n i} \zeta_{i}\right|^{q} I\left(\left|\omega_{n i} \zeta_{i}\right|>W_{n}(p),\left|\zeta_{i}\right| \leq n^{1 / p}\right)\right]}{W_{n}^{p}(p)}
\end{aligned}
$$




$$
\begin{aligned}
\leq & C \sum_{n=1}^{\infty} n^{r-2} \frac{\sum_{i=-\infty}^{\infty}\left|\omega_{n i}\right|^{p} E\left|\zeta_{0}\right|^{p} I\left(\left|\zeta_{0}\right|>n^{1 / p}\right)}{W_{n}^{p}(p)} \\
& +C \sum_{n=1}^{\infty} n^{r-2} \frac{\left(W_{n}(p)\right)^{p-q} \sum_{i=-\infty}^{\infty}\left|\omega_{n i}\right|^{q} E\left|\zeta_{0}\right|^{q} I\left(\left|\zeta_{0}\right| \leq n^{1 / p}\right)}{W_{n}^{p}(p)} \\
= & C \sum_{n=1}^{\infty} n^{r-2} E\left|\zeta_{0}\right|^{p} I\left(\left|\zeta_{0}\right|>n^{1 / p}\right) \\
& +C \sum_{n=1}^{\infty} n^{r-2}\left(\frac{W_{n}(q)}{W_{n}(p)}\right)^{q} E\left|\zeta_{0}\right|^{q} I\left(\left|\zeta_{0}\right| \leq n^{1 / p}\right) \\
\leq & C \sum_{n=1}^{\infty} n^{r-2} E\left|\zeta_{0}\right|^{p} I\left(\left|\zeta_{0}\right|>n^{1 / p}\right)+C \sum_{n=1}^{\infty} n^{r-1-q / p} E\left|\zeta_{0}\right|^{q} I\left(\left|\zeta_{0}\right| \leq n^{1 / p}\right) \\
\leq & C E\left|\zeta_{0}\right|^{r p}<\infty .
\end{aligned}
$$

Thus the first series on the right-hand side of (2.2) converges.

We next prove that the second series on the right-hand side of (2.2) converges. We have by Lemma 2.2 that for $t>2$,

$$
\begin{aligned}
& \sum_{n=1}^{\infty} n^{r-2} P\left(\left|T_{n}^{\prime \prime}\right|>W_{n}(p) \varepsilon / 2\right) \\
& \leq C \sum_{n=1}^{\infty} n^{r-2} \frac{\sum_{i=-\infty}^{\infty} E\left|\omega_{n i} \zeta_{i}\right|^{t} I\left(\left|\omega_{n i} \zeta_{i}\right| \leq W_{n}(p)\right)}{W_{n}^{t}(p)} \\
& \quad+C \sum_{n=1}^{\infty} n^{r-2} \exp \left\{-\frac{\varepsilon^{2} W_{n}^{2}(p)}{2(t+2)^{2} e^{t} \sum_{i=-\infty}^{\infty} \operatorname{Var}\left(\omega_{n i} \zeta_{i} I\left(\left|\omega_{n i} \zeta_{i}\right| \leq W_{n}(p)\right)\right)}\right\} .
\end{aligned}
$$

Hence it is enough to show that two series on the right-hand side of (2.3) converge.

If we take $t>q$, then we have by Lemma 2.3 that

$$
\begin{aligned}
\sum_{n=1}^{\infty} & n^{r-2} \frac{\sum_{i=-\infty}^{\infty} E\left|\omega_{n i} \zeta_{i}\right|^{t} I\left(\left|\omega_{n i} \zeta_{i}\right| \leq W_{n}(p)\right)}{W_{n}^{t}(p)} \\
= & \sum_{n=1}^{\infty} n^{r-2} \frac{\sum_{i=-\infty}^{\infty} E\left|\omega_{n i} \zeta_{i}\right|^{t} I\left(\left|\omega_{n i} \zeta_{i}\right| \leq W_{n}(p),\left|\zeta_{i}\right|>n^{1 / p}\right)}{W_{n}^{t}(p)} \\
& +\sum_{n=1}^{\infty} n^{r-2} \frac{\sum_{i=-\infty}^{\infty} E\left|\omega_{n i} \zeta_{i}\right|^{t} I\left(\left|\omega_{n i} \zeta_{i}\right| \leq W_{n}(p),\left|\zeta_{i}\right| \leq n^{1 / p}\right)}{W_{n}^{t}(p)} \\
= & \sum_{n=1}^{\infty} n^{r-2} \frac{\sum_{i=-\infty}^{\infty} E\left[\left|\omega_{n i} \zeta_{i}\right|^{t-p}\left|\omega_{n i} \zeta_{i}\right|^{p} I\left(\left|\omega_{n i} \zeta_{i}\right| \leq W_{n}(p),\left|\zeta_{i}\right|>n^{1 / p}\right)\right]}{W_{n}^{t}(p)} \\
& +\sum_{n=1}^{\infty} n^{r-2} \frac{\sum_{i=-\infty}^{\infty} E\left[\left|\omega_{n i} \zeta_{i}\right|^{t-q}\left|\omega_{n i} \zeta_{i}\right|^{q} I\left(\left|\omega_{n i} \zeta_{i}\right| \leq W_{n}(p),\left|\zeta_{i}\right| \leq n^{1 / p}\right)\right]}{W_{n}^{t}(p)} \\
\leq & \sum_{n=1}^{\infty} n^{r-2} \frac{\left(W_{n}(p)\right)^{t-p} \sum_{i=-\infty}^{\infty}\left|\omega_{n i}\right|^{p} E\left|\zeta_{0}\right|^{p} I\left(\left|\zeta_{0}\right|>n^{1 / p}\right)}{W_{n}^{t}(p)}
\end{aligned}
$$




$$
\begin{aligned}
& +\sum_{n=1}^{\infty} n^{r-2} \frac{\left(W_{n}(p)\right)^{t-q} \sum_{i=-\infty}^{\infty}\left|\omega_{n i}\right|^{q} E\left|\zeta_{0}\right|^{q} I\left(\left|\zeta_{0}\right| \leq n^{1 / p}\right)}{W_{n}^{t}(p)} \\
= & \sum_{n=1}^{\infty} n^{r-2} E\left|\zeta_{0}\right|^{p} I\left(\left|\zeta_{0}\right|>n^{1 / p}\right)+\sum_{n=1}^{\infty} n^{r-2}\left(\frac{W_{n}(q)}{W_{n}(p)}\right)^{q} E\left|\zeta_{0}\right|^{q} I\left(\left|\zeta_{0}\right| \leq n^{1 / p}\right) \\
\leq & \sum_{n=1}^{\infty} n^{r-2} E\left|\zeta_{0}\right|^{p} I\left(\left|\zeta_{0}\right|>n^{1 / p}\right)+\sum_{n=1}^{\infty} n^{r-1-q / p} E\left|\zeta_{0}\right|^{q} I\left(\left|\zeta_{0}\right| \leq n^{1 / p}\right) \\
\leq & C E\left|\zeta_{0}\right|^{r p}<\infty .
\end{aligned}
$$

Hence the first series on the right-hand side of (2.3) converges.

Finally, we show that the second series on the right-hand side of (2.3) converges. Since $p<s \leq 2$, we have that

$$
\begin{aligned}
& \frac{\sum_{i=-\infty}^{\infty} \operatorname{Var}\left(\omega_{n i} \zeta_{i} I\left(\left|\omega_{n i} \zeta_{i}\right| \leq W_{n}(p)\right)\right)}{W_{n}^{2}(p)} \\
& \quad \leq \frac{\sum_{i=-\infty}^{\infty} E\left|\omega_{n i} \zeta_{i}\right|^{2} I\left(\left|\omega_{n i} \zeta_{i}\right| \leq W_{n}(p)\right)}{W_{n}^{2}(p)} \\
& =\frac{\sum_{i=-\infty}^{\infty} E\left|\omega_{n i} \zeta_{i}\right|^{s+2-s} I\left(\left|\omega_{n i} \zeta_{i}\right| \leq W_{n}(p)\right)}{W_{n}^{2}(p)} \\
& \quad \leq \frac{\left(W_{n}(p)\right)^{2-s} \sum_{i=-\infty}^{\infty} E\left|\omega_{n i} \zeta_{i}\right|^{s}}{W_{n}^{2}(p)} \\
& =\frac{\sum_{i=-\infty}^{\infty}\left|\omega_{n i}\right|^{s} E\left|\zeta_{0}\right|^{s}}{W_{n}^{s}(p)} \\
& =\left(\frac{W_{n}(s)}{W_{n}(p)}\right)^{s} E\left|\zeta_{0}\right|^{s} \\
& =o(1 / \log n),
\end{aligned}
$$

which implies that

$$
\begin{aligned}
& \sum_{n=1}^{\infty} n^{r-2}\left\{-\frac{\varepsilon^{2} W_{n}^{2}(p)}{2(t+2)^{2} e^{t} \sum_{i=-\infty}^{\infty} \operatorname{Var}\left(\omega_{n i} \zeta_{i} I\left(\left|\omega_{n i} \zeta_{i}\right| \leq W_{n}(p)\right)\right)}\right\} \\
& \quad \leq C \sum_{n=1}^{\infty} n^{r-2}\left\{-\frac{\varepsilon^{2} \log n}{2(t+2)^{2} e^{t} o(1)}\right\}<\infty .
\end{aligned}
$$

The next theorem treats the case $r=1$.

Theorem 2.2 Let $1 \leq p<2$. Let $\left\{a_{i}, i \in \mathbb{Z}\right\}$ be a sequence of real numbers with

$$
\sum_{i=-\infty}^{\infty}\left|a_{i}\right|^{\theta}<\infty \quad \text { for some } 0<\theta<p .
$$

Suppose that $\left\{X_{n}, n \geq 1\right\}$ is the linear process of a sequence $\left\{\zeta_{i}, i \in \mathbb{Z}\right\}$ of i.i.d. random variables with mean zero and $E\left|\zeta_{0}\right|^{p}<\infty$. Furthermore, assume that

$$
W_{n}(\theta) / W_{n}(p)=O\left(n^{1 / \theta-1 / p}\right)
$$


and

$$
W_{n}(q) / W_{n}(p)=O\left(n^{1 / q-1 / p}\right) \quad \text { for some } q \in(p, 2)
$$

Then

$$
\sum_{n=1}^{\infty} n^{-1} P\left(\left|\sum_{k=1}^{n} X_{k}\right|>W_{n}(p) \varepsilon\right)<\infty \text { for all } \varepsilon>0 .
$$

Proof The proof is similar to that of Theorem 2.1(1). We proceed with two cases $1 \leq \theta<p$ and $0<\theta<1$.

For the case $1 \leq \theta<p$, we have by Lemmas 2.1 and 2.3 that

$$
\begin{aligned}
\sum_{n=1}^{\infty} n^{-1} P\left(\left|S_{n}^{\prime}\right|>W_{n}(p) \varepsilon / 2\right) & \leq \sum_{n=1}^{\infty} n^{-1} \frac{2^{\theta} E\left|S_{n}^{\prime}\right|^{\theta}}{\varepsilon^{\theta} W_{n}^{\theta}(p)} \\
& \leq C \sum_{n=1}^{\infty} n^{-1} \frac{\sum_{i=-\infty}^{\infty}\left|\omega_{n i}\right|^{\theta} E\left|\zeta_{0}\right|^{\theta} I\left(\left|\zeta_{0}\right|>n^{1 / p}\right)}{W_{n}^{\theta}(p)} \\
& =C \sum_{n=1}^{\infty} n^{-1}\left(\frac{W_{n}(\theta)}{W_{n}(p)}\right)^{\theta} E\left|\zeta_{0}\right|^{p} I\left(\left|\zeta_{0}\right|>n^{1 / p}\right) \\
& \leq C \sum_{n=1}^{\infty} n^{-1} n^{(1 / \theta-1 / p) \theta} E\left|\zeta_{0}\right|^{p} I\left(\left|\zeta_{0}\right|>n^{1 / p}\right) \\
& \leq C E\left|\zeta_{0}\right|^{p}<\infty .
\end{aligned}
$$

As in the proof of Theorem 2.1(1), we have that

$$
\sum_{n=1}^{\infty} n^{-1} P\left(\left|S_{n}^{\prime \prime}\right|>W_{n}(p) \varepsilon / 2\right) \leq C E\left|\zeta_{0}\right|^{p}<\infty
$$

For the case $0<\theta<1$, we rewrite $\sum_{k=1}^{n} X_{k}$ as

$$
\begin{aligned}
\sum_{k=1}^{n} X_{k}= & \sum_{i=-\infty}^{\infty} \omega_{n i} \zeta_{i} I\left(\left|\zeta_{i}\right|>n^{1 / p}\right)+\sum_{i=-\infty}^{\infty} \omega_{n i}\left[\zeta_{i} I\left(\left|\zeta_{i}\right| \leq n^{1 / p}\right)-E \zeta_{i} I\left(\left|\zeta_{i}\right| \leq n^{1 / p}\right)\right] \\
& -\sum_{i=-\infty}^{\infty} \omega_{n i} E \zeta_{i} I\left(\left|\zeta_{i}\right|>n^{1 / p}\right) \\
:= & S_{n}^{\prime}+S_{n}^{\prime \prime}-S_{n}^{\prime \prime \prime}
\end{aligned}
$$

If $0<\theta<1$, then $\sum_{n=1}^{\infty}\left|a_{n}\right| \leq\left(\sum_{n=1}^{\infty}\left|a_{n}\right|^{\theta}\right)^{1 / \theta}<\infty$. It follows by Lemma 2.4 that

$$
\begin{aligned}
W_{n}^{-1}(p)\left|S_{n}^{\prime \prime \prime}\right| & \leq W_{n}^{-1}(p) \sum_{i=-\infty}^{\infty}\left|\omega_{n i}\right| E\left|\zeta_{0}\right| I\left(\left|\zeta_{0}\right|>n^{1 / p}\right) \\
& \leq C n^{1-1 / p} E\left|\zeta_{0}\right| I\left(\left|\zeta_{0}\right|>n^{1 / p}\right) \\
& \leq C E\left|\zeta_{0}\right|^{p} I\left(\left|\zeta_{0}\right|>n^{1 / p}\right) \rightarrow 0
\end{aligned}
$$


as $n \rightarrow \infty$. Hence

$$
\begin{aligned}
& \sum_{n=1}^{\infty} n^{-1} P\left(\left|\sum_{k=1}^{n} X_{k}\right|>W_{n}(p) \varepsilon\right) \\
& \quad \leq C \sum_{n=1}^{\infty} n^{-1} P\left(\left|S_{n}^{\prime}\right|>W_{n}(p) \varepsilon / 3\right)+C \sum_{n=1}^{\infty} n^{-1} P\left(\left|S_{n}^{\prime \prime}\right|>W_{n}(p) \varepsilon / 3\right) .
\end{aligned}
$$

The rest of the proof is the same as that of the previous case and is omitted.

The following corollary extends Theorem 1.1 to the short-range dependent linear processes.

Corollary 2.1 Let $r \geq 1,1 \leq p<2$, and $r p>1$. Let $\left\{a_{i}, i \in \mathbb{Z}\right\}$ be an absolutely summable sequence of real numbers. Suppose that $\left\{X_{n}, n \geq 1\right\}$ is the linear process of a sequence $\left\{\zeta_{i}, i \in\right.$ $\mathbb{Z}\}$ of i.i.d. random variables with mean zero and $E\left|\zeta_{0}\right|^{r p}<\infty$. Then

$$
\sum_{n=1}^{\infty} n^{r-2} P\left(\left|\sum_{k=1}^{n} X_{k}\right|>n^{1 / p} \varepsilon\right)<\infty \quad \text { for all } \varepsilon>0 .
$$

Proof We first note that

$$
\sum_{i=-\infty}^{\infty}\left|a_{i}\right|^{p} \leq\left(\sum_{i=-\infty}^{\infty}\left|a_{i}\right|\right)^{p}<\infty .
$$

If $1<p<2$, then we take $\theta$ such that $1 \leq \theta<p$. Then

$$
\sum_{i=-\infty}^{\infty}\left|a_{i}\right|^{\theta} \leq\left(\sum_{i=-\infty}^{\infty}\left|a_{i}\right|\right)^{\theta}<\infty .
$$

By Lemma 2.4, for any $t>0$, there exist positive constants $C_{1}$ and $C_{2}$ independent of $n$ such that

$$
C_{1} n^{1 / t} \leq W_{n}(t) \leq C_{2} n^{1 / t} \quad \text { for all } n \geq 1 .
$$

Then all conditions on $W_{n}(\cdot)$ in Theorems 2.1 and 2.2 are easily satisfied. Hence the proof follows from Theorems 2.1 and 2.2.

Remark 2.1 In Corollary 2.1, the case $r p=1$ (i.e., $r=1$ and $p=1$ ) is not considered. In fact, Corollary 2.1 does not hold for this case (see Sung [18]).

\section{An estimation of $W_{n}(t)$ for the long-range dependent case}

As we have seen in Sections 1 and 2, it is easy to estimate $W_{n}(t)$ for the short-range dependent case. In this section, we propose a method to estimate $W_{n}(t)$ for the long-range dependent case. It is not easy to estimate $W_{n}(t)$ when the sequence $\left\{a_{i}, i \in \mathbb{Z}\right\}$ is not absolutely summable. For simplicity, we will consider non-increasing sequences of positive numbers. For the finiteness of $W_{n}(t)$, without loss of generality, it is necessary to assume that $a_{i}=0$ if $i \leq 0$ and $\sum_{i=1}^{\infty} a_{i}^{t}<\infty$. 
Lemma 3.1 Let $t>0$. Let $\left\{a_{i}, i \in \mathbb{Z}\right\}$ be a non-increasing sequence of positive real numbers satisfying $a_{i}=0$ if $i \leq 0$ and $\sum_{i=1}^{\infty} a_{i}^{t}<\infty$. Then

$$
\frac{n}{2}\left(a_{1}+\cdots+a_{[n / 2]}\right)^{t}+n^{t} \sum_{i=n}^{\infty} a_{i}^{t} \leq W_{n}^{t}(t) \leq 2 n\left(a_{1}+\cdots+a_{n}\right)^{t}+n^{t} \sum_{i=n}^{\infty} a_{i}^{t} .
$$

Proof Since $a_{i}=0$ if $i \leq 0$ and $0<a_{i} \downarrow$, we get that

$$
\begin{aligned}
W_{n}^{t}(t) & =\sum_{i=1}^{n}\left(\sum_{j=1}^{i} a_{j}\right)^{t}+\sum_{i=1}^{n}\left(\sum_{j=1}^{n} a_{i+j}\right)^{t}+\sum_{i=n+1}^{\infty}\left(\sum_{j=1}^{n} a_{i+j}\right)^{t} \\
& \leq 2 n\left(a_{1}+\cdots+a_{n}\right)^{t}+n^{t} \sum_{i=n+1}^{\infty} a_{i+1}^{t} \\
& \leq 2 n\left(a_{1}+\cdots+a_{n}\right)^{t}+n^{t} \sum_{i=n}^{\infty} a_{i}^{t} .
\end{aligned}
$$

Similarly,

$$
\begin{aligned}
W_{n}^{t}(t) & =\sum_{i=1}^{n-1}\left(\sum_{j=1}^{i} a_{j}\right)^{t}+\sum_{i=1}^{\infty}\left(\sum_{j=0}^{n-1} a_{i+j}\right)^{t} \\
& \geq \sum_{i=[n / 2]}^{n-1}\left(\sum_{j=1}^{i} a_{j}\right)^{t}+n^{t} \sum_{i=n}^{\infty} a_{i}^{t} \\
& \geq \frac{n}{2}\left(a_{1}+\cdots+a_{[n / 2]}\right)^{t}+n^{t} \sum_{i=n}^{\infty} a_{i}^{t} .
\end{aligned}
$$

Thus the proof is completed.

The following lemma can be found in Martikainen [19].

Lemma 3.2 (Martikainen [19]) Let $\left\{b_{n}, n \geq 1\right\}$ be a non-decreasing sequence of positive real numbers. Then

$$
\sum_{i=n}^{\infty} \frac{1}{i b_{i}}=O\left(b_{n}^{-1}\right) \Longleftrightarrow \liminf _{n \rightarrow \infty} \frac{b_{r n}}{b_{n}}>1 \quad \text { for some integer } r \geq 2
$$

Similarly, we can obtain a counterpart of Lemma 3.2.

Lemma 3.3 Let $\left\{b_{n}, n \geq 1\right\}$ be a non-decreasing sequence of positive real numbers. Then

$$
\sum_{i=1}^{n} \frac{b_{i}}{i}=O\left(b_{n}\right) \Longleftrightarrow \liminf _{n \rightarrow \infty} \frac{b_{r n}}{b_{n}}>1 \quad \text { for some integer } r \geq 2 .
$$

Proof The proof is similar to that of Lemma 3.2 and is omitted.

Using Lemmas 3.2 and 3.3, we have the following lemma. 
Lemma 3.4 Let $t>1$ and let $\left\{a_{n}, n \geq 1\right\}$ be a sequence of positive real numbers satisfying $n a_{n} \uparrow, n a_{n}^{t} \downarrow$, and

$$
\frac{1}{r}<\liminf _{n \rightarrow \infty} \frac{a_{r n}}{a_{n}} \leq \limsup _{n \rightarrow \infty} \frac{a_{r n}}{a_{n}}<\left(\frac{1}{r}\right)^{1 / t} \quad \text { for some integer } r \geq 2 .
$$

Then the following statements hold:

(i) $\sum_{i=n}^{\infty} a_{i}^{t}=O\left(n a_{n}^{t}\right)$.

(ii) $\sum_{i=1}^{n} a_{i}=O\left(n a_{n}\right)$.

Proof The proof of (i) follows from Lemma 3.2. The proof of (ii) follows from Lemma 3.3 .

Now we present a method to estimate $W_{n}(t)$ for the long-range dependent case.

Theorem 3.1 Let $t>1$, and let $\left\{a_{n}, n \geq 1\right\}$ be a sequence of positive real numbers satisfying the same conditions as in Lemma 3.4. Then there exist positive constants $C_{1}$ and $C_{2}$ independent of $n$ such that

$$
C_{1} n^{1+t} a_{n}^{t} \leq W_{n}^{t}(t) \leq C_{2} n^{1+t} a_{n}^{t} \quad \text { for all } n \geq 1
$$

where $a_{i}=0$ if $i \leq 0$.

Proof By the condition $n a_{n}^{t} \downarrow$, we have $\left(a_{n+1} / a_{n}\right)^{t} \leq n /(n+1)$, which implies $0<a_{n} \downarrow$. The upper bound of $W_{n}^{t}(t)$ follows by Lemmas 3.1 and 3.4. For the lower bound, we have by $\liminf _{n \rightarrow \infty} a_{r n} / a_{n}>1 / r$ that

$$
a_{r n} / a_{n} \geq 1 / r \quad \text { for all large } n
$$

It follows that for all large $n$

$$
n^{t} \sum_{i=n}^{\infty} a_{i}^{t} \geq n^{t} \sum_{i=n}^{r n} a_{i}^{t} \geq(r-1) n^{1+t} a_{r n}^{t} \geq(r-1) r^{-t} n^{1+t} a_{n}^{t}
$$

Since $0<a_{n} \downarrow$,

$$
n\left(a_{1}+\cdots+a_{[n / 2]}\right)^{t} \geq n[n / 2]^{t} a_{[n / 2]}^{t} \geq n[n / 2]^{t} a_{n}^{t} .
$$

Hence the lower bound follows from Lemma 3.1.

Finally, we give two long-range dependent linear processes.

Example 3.1 Let $a_{i}=1 / i$ if $i \geq 1$ and $a_{i}=0$ if $i \leq 0$. Then the series $\sum_{i=-\infty}^{\infty} a_{i}$ diverges, but $\sum_{i=-\infty}^{\infty} a_{i}^{t}$ converges if $t>1$. Observe that

$$
\ln (n+1) \leq \sum_{i=1}^{n} a_{i} \leq 1+\ln n
$$


If $t>1$, then

$$
\frac{1}{t-1} n^{-t+1} \leq \sum_{i=n}^{\infty} a_{i}^{t} \leq n^{-t}+\frac{1}{t-1} n^{-t+1}
$$

By Lemma 3.1, for any $t>1$, there exist positive constants $C_{1}$ and $C_{2}$ independent of $n$ such that

$$
C_{1} n(\ln n)^{t} \leq W_{n}^{t}(t) \leq C_{2} n(\ln n)^{t} \quad \text { for all } n \geq 2
$$

Let $X_{n}=\sum_{i=-\infty}^{\infty} a_{i+n} \zeta_{i}$ be the long-range dependent linear process of a sequence $\left\{\zeta_{i}\right\}$ of i.i.d. random variables with mean zero and $E\left|\zeta_{0}\right|^{r p}<\infty$, where $r>1$ and $1<p<2$. Then all conditions of Theorem 2.1 are easily satisfied. By Theorem 2.1,

$$
\sum_{n=1}^{\infty} n^{r-2} P\left(\left|\sum_{k=1}^{n} X_{k}\right|>n^{1 / p} \ln n \varepsilon\right)<\infty \quad \text { for all } \varepsilon>0 .
$$

Example 3.2 Let $1<p<2$. Let $a_{i}=1 / i^{d}$ if $i \geq 1$ and $a_{i}=0$ if $i \leq 0$, where $1 / p<d<1$. Then the series $\sum_{i=-\infty}^{\infty} a_{i}$ diverges, but $\sum_{i=-\infty}^{\infty} a_{i}^{t}$ converges if $t>1 / d$. Since $a_{2 n} / a_{n}=2^{-d}$, we have by Theorem 3.1 that

$$
C_{1} n^{1+t-d t} \leq W_{n}^{t}(t) \leq C_{2} n^{1+t-d t} \quad \text { for all } n \geq 1
$$

Let $X_{n}=\sum_{i=-\infty}^{\infty} a_{i+n} \zeta_{i}$ be the long-range dependent linear process of a sequence $\left\{\zeta_{i}\right\}$ of i.i.d. random variables with mean zero and $E\left|\zeta_{0}\right|^{p}<\infty$. Take $\theta$ such that $1 / d<\theta<p$. Then all conditions of Theorem 2.2 are easily satisfied. By Theorem 2.2,

$$
\sum_{n=1}^{\infty} n^{-1} P\left(\left|\sum_{k=1}^{n} X_{k}\right|>n^{1 / p+1-d} \varepsilon\right)<\infty \quad \text { for all } \varepsilon>0 .
$$

\section{Acknowledgements}

The research of Pingyan Chen is supported by the National Natural Science Foundation of China (No. 11271161). The research of Soo Hak Sung is supported by Basic Science Research Program through the National Research Foundation of Korea (NRF) funded by the Ministry of Education (2017R1D1A1B03029898).

\section{Competing interests}

The authors declare that they have no competing interests.

\section{Authors' contributions}

All authors read and approved the manuscript.

\section{Author details}

'Department of Statistics, Jinan University, Guangzhou, 510630, P.R. China. ${ }^{2}$ Department of Mathematics, Jinan University, Guangzhou, 510630, P.R. China. ${ }^{3}$ Department of Applied Mathematics, Pai Chai University, Daejeon, 35345, South Korea.

\section{Publisher's Note}

Springer Nature remains neutral with regard to jurisdictional claims in published maps and institutional affiliations. 
References

1. Baum, LE, Katz, M: Convergence rates in the law of large numbers. Trans. Am. Math. Soc. 120, 108-123 (1965)

2. Alf, C: Rates of convergence for the laws of large numbers for independent Banach-valued random variables J. Multivar. Anal. 5, 322-329 (1975)

3. Alsmeyer, G: Convergence rates in the law of large numbers for martingales. Stoch. Process. Appl. 36, 181-194 (1990)

4. Heyde, CC, Rohatgi, VK: A pair of complementary theorems on convergence rates in the law of large numbers. Proc. Camb. Philol. Soc. 63, 73-82 (1967)

5. Hu, T-C, Weber, NC: On the rate of convergence in the strong law of large numbers for arrays. Bull. Aust. Math. Soc. 45 479-482 (1992)

6. Rohatgi, VK: Convergence rates in the law of large numbers II. Proc. Camb. Philol. Soc. 64, 485-488 (1968)

7. Hsu, PL, Robbins, H: Complete convergence and the law of large numbers. Proc. Natl. Acad. Sci. USA 33, 25-31 (1947)

8. Katz, M: The probability in the tail of a distribution. Ann. Math. Stat. 34, 312-318 (1963)

9. Giraitis, L, Koul, HL, Surgailis, D: Large Sample Inference for Long Memory Processes. Imperial College Press, London (2012)

10. Koopmans, LH: An exponential bound on the strong law of large numbers for linear stochastic processes with absolutely convergent coefficients. Ann. Math. Stat. 32, 583-586 (1961)

11. Hanson, DL, Koopmans, LH: On the convergence rate of the law of large numbers for linear combinations of independent random variables. Ann. Math. Stat. 36, 559-564 (1965)

12. Li, D, Rao, MB, Wang, X: Complete convergence of moving average processes. Stat. Probab. Lett. 14, 111-114 (1992)

13. Zhang, L: Complete convergence of moving average processes under dependence assumptions. Stat. Probab. Lett. 30, 165-170 (1996)

14. Characiejus, V, Račkauskas, A: Weak law of large numbers for linear processes. Acta Math. Hung. 149, 215-232 (2016)

15. Burton, RM, Dehling, H: Large deviations for some weakly dependent random processes. Stat. Probab. Lett. 9, 397-401 (1990)

16. von Bahr, B, Esseen, CG: Inequalities for the $r$ th absolute moment of a sum of random variables, $1 \leq r \leq 2$. Ann. Math. Stat. 36, 299-303 (1965)

17. Nagaev, SV: Large deviations of sums of independent random variables. Ann. Probab. 7, 745-789 (1979)

18. Sung, SH: A note on the complete convergence of moving average processes. Stat. Probab. Lett. 79, 1387-1390 (2009)

19. Martikainen, Al: Criteria for strong convergence of normalized sums of independent random variables and their applications. Theory Probab. Appl. 29, 519-533 (1985)

\section{Submit your manuscript to a SpringerOpen ${ }^{\circ}$ journal and benefit from:}

- Convenient online submission

- Rigorous peer review

Open access: articles freely available online

- High visibility within the field

- Retaining the copyright to your article

Submit your next manuscript at $>$ springeropen.com 